\title{
Observation of cross sectional semiconductor sample with newly developed SEI/STEM/TEM microscope
}

\author{
N. Endo, ${ }^{*}$ T. Kuba, ${ }^{*}$ T. Suzuki, ${ }^{*}$ E. Okunishi, ${ }^{*}$ and M. Kersker ${ }^{* *}$
}

*Application and Research Center, JEOL Ltd., 3-1-2 Musashino, Akishima, Tokyo, 196-8558, Japan

**JEOL USA, Inc. 11 Dearborn Rd. Peabody, MA 01960

Recently, semiconductor analysis method is roughly divided into two ways. One way is nano-structural analysis of defects or dislocations using thin films less than $100 \mathrm{~nm}$ in thickness, the other way is stereo observation or tomography of via structures or voids using thicker samples. It is important that the above-mentioned information with one microscope can be obtained. Here, we report nano-structural analysis and stereo observation of the cross sectional semiconductor sample using a newly developed nano-analysis electron microscope (JEM-2500SE), which is equipped with field emission gun (FEG) and dry vacuum system. [1]

The microscope enables us to observe SEI, STEM-BF image, STEM-DF image, TEM image, diffraction pattern and elemental map images of a sample in a bright room with easy-to-use operations controlled by personal computer, since TV cameras for TEM images and a digital scanning device with SEI, BFI and DFI detectors for scanning images are all built in the microscope. These images are digitized and displayed on a PC monitor and are saved in digital PC format (BITMAP, JPEG or TIFF).

Stereo observation of SEI using a bulk sample is effective, which is shown in Fig.1, because SEI at $200 \mathrm{kV}$ accelerating voltage offers 3D information on the specimen. Moreover, stereo observation of STEM is more effective using a sample thickness of 1um.

The nano-structural information is obtained from the high resolution and high magnification images, which can be obtained in both TEM and STEM modes. The resolutions in both modes are $0.14 \mathrm{~nm}$ in TEM mode, and $0.2 \mathrm{~nm}$ in STEM mode. The lattice in a TEM image can be quickly obtained because of absence of beam scan, and that in a STEM-BF and STEM-DF images, which are shown in Figs. 2 and 3, have advantages of high contrast controlled electronically and good match in the field of view with that in elemental map in analysis mode.

We believe that the microscope is the most suitable analytical tool to observe a semiconductor cross-sectional sample. 


\section{References}

[1] Frank, J., Electron Tomography, Plenum Press, New York and London, 1992

(a)

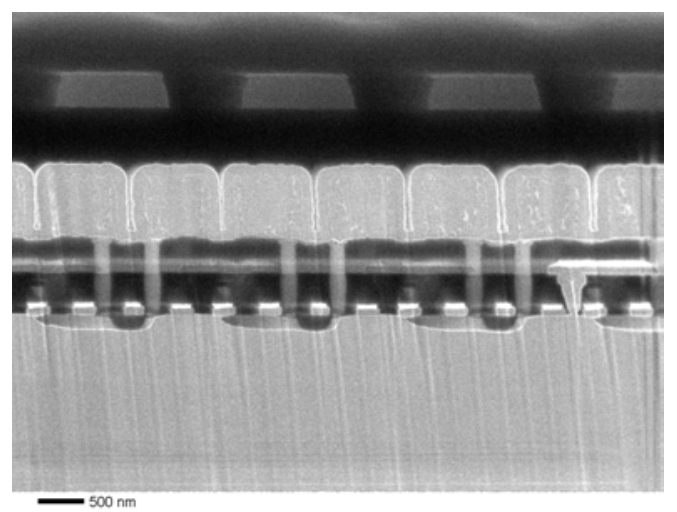

(b)

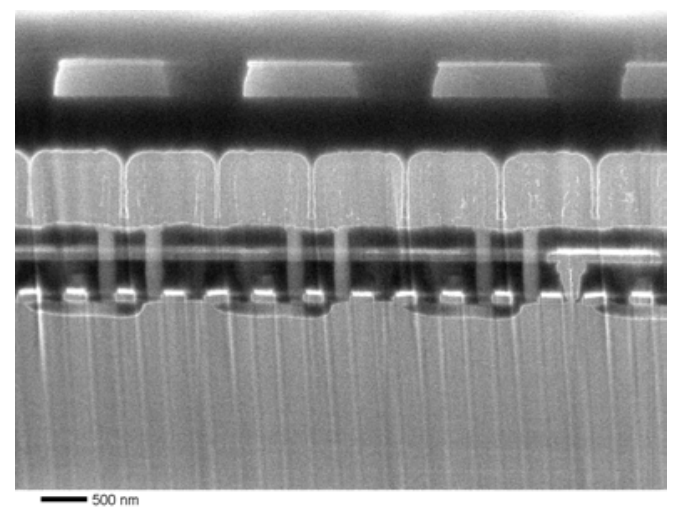

Fig.1 Stereo observation of SEI taken at a tilt angle of +30 degrees (a) and -30 degrees (b).

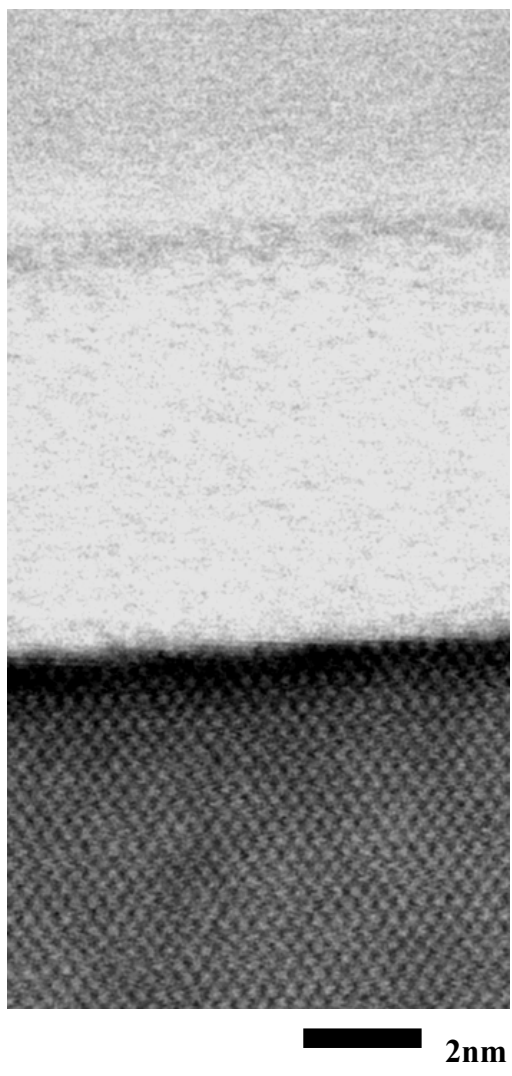

FIG.2 High-resolution STEM-BF image of a device cross-sectional thin film.
Poly-Si

$\mathrm{SiO}_{2}$

Si

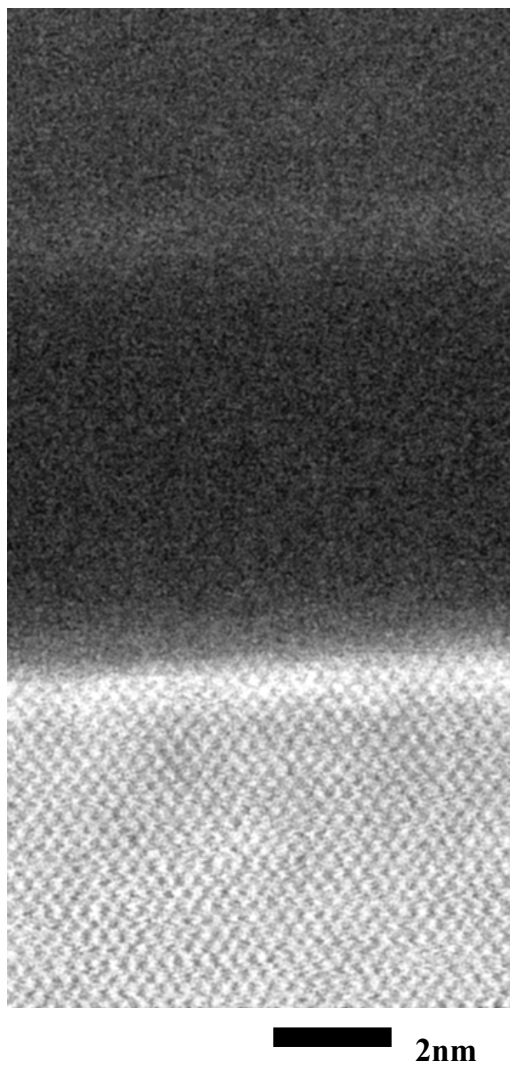

FIG.3 High-resolution STEM-HAADF image of a device cross-sectional thin film. 